\title{
EchoGéo
}

\section{Epizooties et géographie du commerce du bétail dans la Corne d'Afrique}

La guerre des quarantaines dans la région nord-somali

\section{Géraldine Pinauldt}

\section{(2) OpenEdition}

\section{Journals}

\section{Édition électronique}

URL : https://journals.openedition.org/echogeo/11021

DOI : 10.4000/echogeo. 11021

ISSN : 1963-1197

Éditeur

Pôle de recherche pour l'organisation et la diffusion de l'information géographique (CNRS UMR 8586)

Référence électronique

Géraldine Pinauldt, «Epizooties et géographie du commerce du bétail dans la Corne d'Afrique », EchoGéo [En ligne], 8| 2009, mis en ligne le 19 mars 2009, consulté le 01 août 2021. URL : http:// journals.openedition.org/echogeo/11021; DOI : https://doi.org/10.4000/echogeo.11021

Ce document a été généré automatiquement le 1 août 2021.

EchoGéo est mis à disposition selon les termes de la licence Creative Commons Attribution - Pas d'Utilisation Commerciale - Pas de Modification 4.0 International (CC BY-NC-ND) 


\title{
Epizooties et géographie du commerce du bétail dans la Corne d'Afrique
}

La guerre des quarantaines dans la région nord-somali

\author{
Géraldine Pinauldt
}

1 En 1997-1998, l'Afrique de l'Est et la Corne de l'Afrique connaissent l'un des plus graves épisodes humain de fièvre de la vallée du Rifti. En 2000, pour la première fois depuis l'identification de la maladie en 1931, un foyer humain se déclare hors de l'Afrique, en Arabie Saoudite et au Yémen, principales destinations des exportations du bétail estafricain. Ces deux épisodes ont été à l'origine d'embargos sur le bétail de la région, touchant en son cœur un système commercial régional dont les revenus sont essentiels à la fois pour les États et pour leurs habitants. Il est particulièrement intéressant d'étudier l'impact de la fièvre de la vallée du Rift dans la grande région somali, qui, en plus du territoire de la Somalie, comprend le nord-est du Kenya, l'est de l'Éthiopie et une partie du territoire djiboutien. Les Somali dominent en effet ce commerce d'exportation du bétail vers la péninsule arabe depuis la fin du $19^{\mathrm{e}}$ siècle, le système s'étant extraverti avec la mise en place d'un protectorat britannique destiné à approvisionner les garnisons anglaises d'Aden. Depuis, les exportations de bétail se sont intensifiées à destination des pays de la péninsule arabe et, plus que dans d'autres sociétés pastorales voisines, une classe marchande spécialisée dans l'exportation de bétail s'est mise en place. Environ $60 \%$ de la population de la grande région somali dépend de l'élevage.

2 Depuis les années 1980 cependant, le bétail Somali est en concurrence avec des importations d'Europe de l'Est, de Syrie, d'Amérique latine, d'Australie et de Nouvelle Zélande. La plus grande ouverture de ce marché se traduit également par le renforcement d'une gouvernance sanitaire mondiale et l'alignement sur des normes sanitaires. Elle a pour double objectif de protéger la santé des consommateurs et de tenter de sécuriser les marchés des pays exportateurs, en les assistant dans la mise en place de services de certification efficaces. Dans le cadre de la crise de la fièvre de la 
vallée du Rift dans la Corne de l'Afrique, la mise aux normes sanitaires passe entre autre par l'établissement de centres de quarantaine modernes, pourvus de services vétérinaires, de laboratoire d'analyse, et reconnus dans le cadre d'accords bilatéraux par les pays importateurs, souvent avec la coordination d'institutions internationales telles que l'Organisation Internationale de la Santé Animale (OIE).

$\mathrm{Du}$ fait de cet épisode de fièvre de la vallée du Rift en 2000, le bétail de la région (toutes espèces confondues) va être l'objet d'embargos successifs. En effet, les pays arabes, parmi lesquels l'Arabie Saoudite accueille chaque année des millions de pèlerins, ne souhaitent pas prendre le risque d'une nouvelle épidémie sur leur sol. L'embargo n'a pu être officiellement levé -bien que partiellement- qu'avec l'ouverture à Djibouti en 2006 d'un centre de quarantaine permettant de certifier tout le bétail de la région destiné à être exporté vers les pays du Golfe.

4 Le découpage de cette région en plusieurs entités politiques distinctes place le commerce de bétail sous des juridictions différentes, le soumettant aux frontières et aux jeux géopolitiques à l'échelle de la Corne de l'Afrique. En effet, les trois principaux ports d'exportation d'animaux vivants sont situés dans trois États distincts. Sur les ruines de l'ancienne Somalie (telle qu'elle apparaît encore sur les atlas), le Somaliland, ou nord-ouest de la Somalie, ancien protectorat britannique, est un territoire indépendant depuis 1991 bien que non encore reconnu. Son principal port est Berbera. Le Puntland, au nord-est, s'est également distingué du reste de la Somalie par une administration autonome, tout en n'affichant pas de volonté séparatiste. Son port est Bosaaso (pour une description précise de la formation de ces nouvelles entités, voir Lewis, 2008). Enfin, Djibouti, ancien territoire français, possède le port le plus important de cette portion de côte, et joue également un rôle dans l'exportation du bétail de la Corne. Le bétail est quant à lui dispersé sur tout l'arrière-pays, principalement dans les basses terres semi-arides de la région somali déjà citées. L'approvisionnement pour l'export peut s'effectuer jusqu'en Ouganda et au Sud Soudan.

5 Ces territoires ont tous connu des bouleversements politiques majeurs depuis les années 1990. En Éthiopie, la chute du régime totalitaire de Mengistu a donné naissance en 1994 à un régime prônant l'ethnofédéralisme et une relative ouverture économique. En Somalie en 1991, la fin de la dictature, de Syad Barre a été suivie de la faillite de l'État au Sud et la (re)naissance d'un État, le Somaliland, au nord-ouest. Enfin, Djibouti vit, depuis la fin des années 1990, un changement d'orientation économique qui a accompagné l'arrivée du second président du pays, traduit notamment par un investissement important venant de la péninsule arabe.

6 Au cours des années 1990 chaque pays découvre ou redécouvre l'importance des bénéfices à réaliser sur ce commerce de bétail à destination de la péninsule arabe. L'obligation de certification de la part des pays importateurs consécutive à l'épidémie bouleverse la hiérarchie des ports d'exportation, aboutissant à une compétition entre les pays exportateurs pour l'établissement de centres de quarantaines reconnus, désormais sésame de l'exportation légale vers les pays arabes. Ces évènements ont également eu pour conséquence la réduction des possibilités d'exportation informelle, forme auparavant dominante. Concordant avec des facteurs internes à chaque État, ils tendent à rallier une partie des commerçants au commerce légal. Ceci reste bien sûr une tendance, qui doit encore être confirmée, et il faut garder à l'esprit que des changements de stratégies des acteurs privés peuvent intervenir très rapidement dans 
l'instabilité généralisée des milieux semi-arides, auxquels appartient la zone d'étude (Holzmann et Kulibaba, 1999). Les recompositions qui vont s'opérer vont ainsi être, entre autres facteurs et dans un premier temps les résultats du jeu des intérêts divergents entre acteurs publics qui souhaitent récupérer des dividendes dans ce commerce et négociants privés qui vont chercher à conserver la liberté de marché que l'informalité leur offrait.

7 La crise sanitaire qui a secoué la région, en amont comme en aval du commerce de bétail, a donné le point de départ à une série de reconfigurations des réseaux commerçants, accompagnées de transformations en profondeur des modes de gestion, dont les effets sur les infrastructures ont des conséquences certaines sur l'organisation spatiale des populations pastorales.

8 Cet article se place dans le cadre d'un travail de thèse dont l'objet est de saisir les recompositions territoriales et géopolitiques provoquées par la mondialisation du commerce d'exportation de bétail dans les régions somali d'Éthiopie, de Djibouti et du Somaliland. La présente étude aborde la concurrence entre les ports d'exportation de bétail dans le contexte de réseaux commerciaux transfrontaliers: Berbera pour le Somaliland, Bosaaso au Puntland et le port de Djibouti, et ses conséquences au niveau des États et dans les arrières-pays. L'objectif est d'analyser la manière dont une crise sanitaire régionale peut amorcer ou accélérer la transformation d'un système commercial. Il est ici question de s'intéresser en particulier aux impacts indirects de ce processus. La convergence des deux contraintes que représentent concurrence nouvelle et normalisation aboutit du côté des importateurs à une instrumentalisation du danger sanitaire et du côté des exportateurs à une concurrence locale transformée.

Les éléments présentés ici sont issus d'un premier travail de terrain en Éthiopie et à Djibouti entre mai et août 2008. Le commerce de bétail dans la région y était alors perturbé par un conflit opposant une partie de l'État somalilandais à un large groupe d'exportateurs. Le matériel, statistique et qualitatif que j'y ai recueilli auprès de commerçants, des services de l'élevage et d'observations dans les deux pays, m'ont permis de replacer cette actualité omniprésente dans son contexte plus large, tentant de saisir les transformations auxquelles elle participe.

10 Afin de mettre en lien les observations réalisées sur le terrain avec les évènements récents ayant touché le commerce d'exportation de bétail dans la zone somali, je commencerai par présenter de manière chronologique l'évolution des exportations dans chaque port à la suite de l'embargo et en Éthiopie au cours des années 2000, et dans un second temps les tentatives de chacun d'établir et de faire reconnaître un centre de quarantaine pour récupérer le marché. Une troisième partie aura pour objet l'interprétation des transformations dans les réseaux marchands et dans les infrastructures qui découlent des aspects étudiés dans les deux premières.

\section{L'impact des embargos sur les exportations}

11 Dans un premier temps, je propose d'observer les variations des exportations des pays concernés par cette étude depuis les années 1990. Ceci permettra d'appréhender les tendances de la redistribution des flux d'exportation entre les différents ports. Depuis maintenant près de 10 ans en effet, on assiste à des " coups de théâtre » qui viennent s'additionner à l'instabilité chronique depuis plus d'un siècle (Djama, 1999). Zoonose, 
embargo, création du centre, ces évènements dépassent l'anecdote: ils sont l'occasion d'une réorganisation des lieux de l'exportation.

Marcel Djama (Bourgeot,1999) a rappelé le profond déséquilibre qui s'est installé dans le système pastoral nord somali pendant la période coloniale. La fin du $19^{\mathrm{e}}$ siècle a marqué l'abandon des exportations de produits issus de la chasse au profit du bétail, et dans un premier temps surtout des petits ruminants. Le volume commercialisé a augmenté tout au long du $20^{\mathrm{e}}$ siècle, avec la multiplication par $7 \mathrm{du}$ nombre d'animaux exportés entre le début et le milieu du siècle. Les Britanniques ayant initié ce commerce, le port de Berbera resta longtemps le débouché majeur (sinon le seul) du bétail de la Corne à destination de la péninsule arabe.

\begin{tabular}{|l|c|}
\hline & $\begin{array}{c}\text { Petits ruminants } \\
\text { (par tehes) }\end{array}$ \\
\hline 1901 Berbera & 63000 \\
\hline 1926 Berbera & 126280 \\
\hline 1959 Berbera & 455000 \\
\hline $\begin{array}{l}\text { 1970-1987, mayenne } \\
\text { annuelle (Berthera+Bosaaso) }\end{array}$ & 1200000 \\
\hline
\end{tabular}

13 Jusqu'à la fin des années 1980, Djibouti, excentré par rapport aux lieux de l'exportation (tous situés en Somalie), était un acteur mineur du commerce de bétail. En 1988, la guerre civile touchant le nord de la Somalie paralysa les exportations depuis Berbera. Djibouti bénéficia de la déviation des exportations et participa pour la première fois de manière importante à l'exportation de bétail. Ce fut une courte parenthèse, puisque en 1991, avec la chute du régime de Syad Barré et l'avènement de la République du Somaliland, les exportations reprirent depuis le port de Berbera, à un niveau supérieur à celui d'avant guerre, avec une moyenne de 2,5 millions de têtes exportées par an entre 1994 et 1997 (FAO-FSAU) pour le seul port de Berbera, et environ 500000 têtes annuelles pour le port de Bosaaso. Le premier embargo, en 1998, à la suite de l'épisode de fièvre de la vallée du Rift, affecte les exportations mais il n'est pas appliqué par tous les pays importateurs. Le volume des exportations est presque divisé de moitié pour Berbera, alors que Bosaaso ne semble pas concerné, ses exportations augmentant légèrement chaque année. Djibouti est toujours hors jeu. Pour l'Éthiopie, l'importance du commerce informel rend difficile le décompte réel des exportations d'animaux vivants.

En 2001, après l'apparition de cas avérés de fièvre de la vallée du Rift en Arabie Saoudite et au Yémen (environ 150 décès humains dans chaque pays), les exportations s'effondrent de l'autre côté du golfe d'Aden, avec une année quasiment nulle pour Berbera et une légère baisse pour Bosaaso.

L'année 2001 marque le début des recompositions régionales: l'embargo instauré par l'ensemble des pays de la péninsule arabe entraîne une inversion des lieux importants du commerce. Le port de Berbera est durablement affecté par la crise. Bosaaso prend sa place comme le principal débouché de la région, alors que le rôle émergent de Djibouti est conforté en 2004 par le choix conjoint de l'Agence des États-Unis pour le Développement International (USAID) et de l'OIE d'y établir un centre d'exportation régional de bétail (ou centre de quarantaine de Djibouti). En novembre 2006, l'établissement anticipe son ouverture prévue à l'origine deux mois plus tard en raison d'un risque de pénurie de moutons pendant la période de pèlerinage en Arabie Saoudite (5 jours pendant lesquels un petit ruminant, la plupart du temps un mouton, est sacrifié 
par pèlerin, alors que plusieurs millions de pèlerins affluent chaque année), lié à une augmentation de prix du bétail australien. Face à une demande pressante, en seulement 15 jours, 250000 moutons et chèvres sont testés et vaccinés dans le nouveau centre. L'ouverture s'effectue ainsi avec des critères de quarantaine revus à la baisse. A partir de cette date, l'Arabie Saoudite, les Émirats Arabes Unis, Oman, le Yémen exigent le passage par la quarantaine de Djibouti de tout le bétail exporté depuis la Corne, affectant surtout le port de Berbera. Ce dernier a subi une perte durable et, depuis 10 ans, n'a pas réussi à regagner son niveau antérieur: avec 1,1 millions de têtes exportées au total en 2006, le niveau des années 1980 n'est même pas atteint, alors que le total des exportations de la Corne est revenu au niveau acceptable de 3,5 millions de têtes annuelles. Ce chiffre doit néanmoins être considéré avec prudence pour deux raisons. La première est le commerce informel, qui demeure important en dépit d'une entrée dans la légalité encouragée par l'obligation du passage en quarantaine. La seconde réside en le risque de double compte dû au flou du statut de Djibouti: ni importateur (le bétail entrant n'est pas considéré comme importé) ni producteur de bétail, ni véritablement exportateur, le bétail en transit exporté par Djibouti peut ainsi avoir été déjà enregistré par les douanes éthiopienne et somalilandaise ou ne pas l'avoir été.

L'Éthiopie est dans une situation différente : en effet, enclavée, elle ne participe pas directement à l'exportation par bateaux du bétail, mais est un producteur important. Bien que le port de Djibouti ait largement bénéficié des mauvaises relations entre l'Éthiopie et l'Érythrée, de la fermeture d'Assab, et de la redirection des flux d'import export vers Djibouti, le commerce de bétail ne suit pas les mêmes routes commerciales que les autres produits exportés par l'Éthiopie. En effet, le bétail éthiopien destiné aux pays arabes vient, pour sa grande majorité, de la région somali, et ses opérateurs privés sont également somali, s'insérant dans les réseaux transnationaux beaucoup plus que dans les réseaux sociaux et commerciaux éthiopiens. Ainsi, l'État éthiopien, en périphérie des zones d'exportation et des principaux lieux de négociation, est pourtant concerné par les recompositions qui suivent les embargos. Un simple regard sur l'évolution des exportations légales (donc comptabilisées) est éloquent: la part des produits de l'élevage, et plus particulièrement de l'exportation de bétail est croissante en Éthiopie : la part des exportations d'animaux vivants dans les exportations éthiopiennes a été multipliée par 6 entre 2003 et 2006, et cette catégorie est passée du $19^{\mathrm{e}}$ au $7^{\mathrm{e}}$ rang des exportations éthiopiennes, contribuant ainsi à $2,7 \%$ des revenus des exportations. Ce produit a connu, de loin, la plus forte croissance à l'exportation sur l'année 2004/2005 (calendrier éthiopien ${ }^{\mathrm{ii}}$ ) avec $456 \%$; les plus fortes croissances d'exportation la même année sont le bétail et les sous produits de l'élevage (cuir, peaux et viande) (Ethiopian Customs authority, 2006).Le revenu issu des exportations de bétail a été multiplié par 12 entre 2003/2004 et 2005/2006 (avant même la réouverture via Djibouti des exportations vers l'Arabie Saoudite), passant de 20 à 240 millions de birr éthiopiens. Cette évolution traduit, au-delà d'une véritable augmentation de la vente de bétail éthiopien, une très progressive légalisation de ce commerce. Elle n'est cependant pas le seul fruit des conséquences des épisodes de fièvre de la vallée du Rift. Elle concorde également avec la progressive et relative ouverture économique amorcée depuis les années 1990 et n'est pas sans lien avec un positionnement progressif de certains notables de la région somali (parmi eux des négociants de bétail) en faveur de l'intégration à l'Éthiopie (jusque là plus supposée que réelle). 
17 Aucun pays importateur n'a pour l'instant établi d'accord reconnaissant une efficacité sanitaire au Somaliland. C'est justement ce point qui crée les tensions actuelles: l'accès à Djibouti rallonge le temps et le coût entre l'achat au producteur et la vente finale au commerçant saoudien, et réveille une compétition entre les ports d'exportation. Posséder son propre centre de quarantaine agréé permet d'exporter directement le bétail du pays, sans passer par Djibouti. Ainsi, chaque État tente d'installer et de faire reconnaître un centre de quarantaine sur son territoire national. Si les investissements réalisés à Djibouti semblent satisfaire pour l'instant les conditions des pays importateurs, le «simple» fait de se doter d'une telle infrastructure ne suffit pas encore à une levée de l'embargo, qui pèse toujours sur le bétail de la Corne ne passant pas à Djibouti.

\section{La guerre des quarantaines}

Depuis la crise sanitaire, les projets de quarantaine se sont ainsi multipliés dans la région : l'Éthiopie avec Dire Dawa et un projet à la frontière djiboutienne, le Puntland avec Bosaaso et le Somaliland avec Berbera ont désormais leur propre quarantaine, financée par des bailleurs publics internationaux comme à Dire-Dawa, ou par des commerçant saoudiens qui accompagnent ces investissements de contrats suscitant le scepticisme d'un certain nombre d'acteurs, comme à Bosaaso et Berbera. Les tentatives d'amélioration de l'accès aux marchés internationaux ne sont pas nouvelles, mais il est certain que cet épisode de fièvre de la vallée du Rift et l'attention renforcée des pays importateurs sur la santé du bétail de la Corne a engagé chaque pays dans une course au renforcement de ses services et infrastructures vétérinaires. Épizootie à part, les crises de 1998 et 2000 interviennent moins d'une dizaine d'année après des changements radicaux dans les administrations éthiopienne et somalienne. Même si ce n'est pas l'objet de cet article de les étudier en profondeur, il est nécessaire de relever ces changements.

19 Le choix - par une commission réunissant l'Union Africaine et l'USAID - d'établir à Djibouti un centre régional d'exportation, ou quarantaine régionale, a ouvert une course à l'ouverture et à la reconnaissance des quarantaines dans la région. Choisi pour sa situation dans une zone exempte de zoonose, et d'une relative stabilité politique, Djibouti est devenu le passage obligatoire pour tout le bétail exporté depuis la Somalie et l'Éthiopie. Une délégation du ministère saoudien de l'agriculture visite les installations tous les trois mois pour renouveler son agrément.

Construit en 2004 avec les financements de l'USAID qui s'est depuis retiré du projet, le centre a été racheté en 2005 par une compagnie privée saoudienne possédant des abattoirs importants en Arabie Saoudite, mais relativement novice dans l'achat de bétail à l'étranger. Cette entreprise a poursuivi les travaux et le gère aujourd'hui. L'ouverture du marché saoudien par l'intermédiaire de la quarantaine de Djibouti crée de nouveaux enjeux.

21 Le commerce s'est dirigé massivement vers Djibouti qui n'était pas à l'origine un important port d'exportation du bétail. L'adhésion tardive de Djibouti à l'OIE, en 2003, témoigne du peu d'intérêt que portait auparavant le pays à ce négoce. Pour Djibouti, l'ouverture de la quarantaine ne revêt pas la même importance économique que pour le Somaliland. En revanche, elle participe d'une stratégie générale visant à placer le pays au cœur du système économique régional, et à se positionner comme interface 
privilégiée entre l'arrière pays de la Corne de l'Afrique (et à terme les flux du COMESA), et les pays de la péninsule arabe ${ }^{1}$. La reconnaissance du Somaliland par la communauté internationale pourrait venir contrarier ce projet djiboutien.

Pour l'instant, l'absence d'un État reconnu au Somaliland l'a empêché d'accueillir la quarantaine régionale. Malgré ses efforts dans la construction de relations commerciales et diplomatiques avec ses voisins, cette entité politique n'est toujours considérée par les voies officielles que comme partie de l'État somalien défaillant. Ainsi, le Somaliland est directement victime du caractère non officiel de son existence, qui, malgré une diplomatie active, est une contrainte majeure à l'implantation de telles infrastructures qui, plus que nationales, se situent au centre d'une coordination régionale. On l'a vu, le pays a subi une baisse importante de ses exportations de bétail, ce marché représentant $80 \%$ des revenus du pays en 2006. Cette importance économique revêt un caractère très politique qui peut, dans le cadre des rapports avec ses voisins, se résumer à une « diplomatie du mouton » qui en dit long sur l'importance symbolique de ce commerce.

La fermeture de la représentation somalilandaise à Djibouti après la décision d'ouverture, en 2005, du centre de quarantaine marque cette déception et à ce jour malgré un réchauffement avéré des relations la représentation n'a pas rouvert. Dans le même ordre d'idée, le geste symbolique de la Chambre de commerce somalilandaise est une illustration intéressante du rôle majeur que le pays souhaite continuer à jouer dans l'exportation de bétail: en soutien à l'armée djiboutienne lors de l'évènement frontalier avec l'Érythrée l'été dernier, le Somaliland a offert 2000 moutons "Berberawi » à Djibouti lors d'une cérémonie au Centre régional d'exportation de bétail, marquant d'un même coup sa crédibilité en tant qu'État souverain et sa mainmise sur la ressource en bétail, bétail nommé d'après sa région d'exportation privilégiée, donc, diront certains, d'origine.

Mais ce jeune État ne peut abandonner cette ressource, et l'accession à un commerce directe avec les pays importateurs est une priorité. Ainsi, le désir d'accès au marché normalisé, et la convergence des intérêts avec des importateurs privés, eux-mêmes soumis à une intense concurrence, a amené l'État somalilandais à conclure un accord avec un important importateur saoudien de bétail. Cet accord offrait le monopole de l'exportation à cet importateur, qui en échange investissait dans la construction d'un centre d'exportation doté d'une quarantaine, prévoyant sa reconnaissance rapide par les services du ministère saoudien de l'agriculture. Pour l'État, il s'agissait d'être en mesure de garantir la santé du bétail exporté, et indirectement, se voir donc reconnaître une légitimité dans sa capacité à produire des services sanitaires efficaces. Il s'agissait également d'offrir au pays une quarantaine permettant de récupérer le flux détourné vers Djibouti, et ainsi les revenus d'exportation qui en découlent. Provoquant l'ire d'une grande partie des exportateurs somali, qui ont vu là une tentative de réduire leurs marges de manœuvre, et d'une certaine manière, la souplesse de leur système "d'adaptation opportuniste", cet accord a abouti au blocage des exportations par Berbera plusieurs semaines en plusieurs occasions. Les commerçants engagés contre ce processus le qualifient d'anticonstitutionnel, la protection du libre échange étant inscrite dans le préambule de la Constitution somalilandaise, et de haraam, soit illégal ou illicite en arabe, puisqu'instaurant un monopole. On voit que pour le Somaliland, les conséquences des récentes épidémies ont été importantes. 

par l'USAID qui, dans le même temps, investissait dans celle de Djibouti. Du côté
éthiopien, on reproche à Djibouti l'obligation pour les animaux éthiopiens d'être
retenus une seconde fois dans la quarantaine de Djibouti. Pourtant, si le passage par la
quarantaine de Djibouti est obligatoire pour les animaux venant d'Éthiopie, qu'ils aient
ou non observés une quarantaine imposée par les autorités éthiopiennes à Dire Dawa,
c'est du seul fait des pays arabes importateurs et non de Djibouti. Les autorités
éthiopiennes exigent le passage par la quarantaine de Dire Dawa pour les animaux
quittant le pays, mais les pays importateurs, à l'exception de l'Égypte, ne la
reconnaissent pas. Le bétail est donc censé être retenu successivement dans deux
quarantaines, même si dans les faits, leur contournement est aisé.

\section{Contraintes sanitaires pour l'exportation : les quarantaines, sésame vers les marchés arabes}
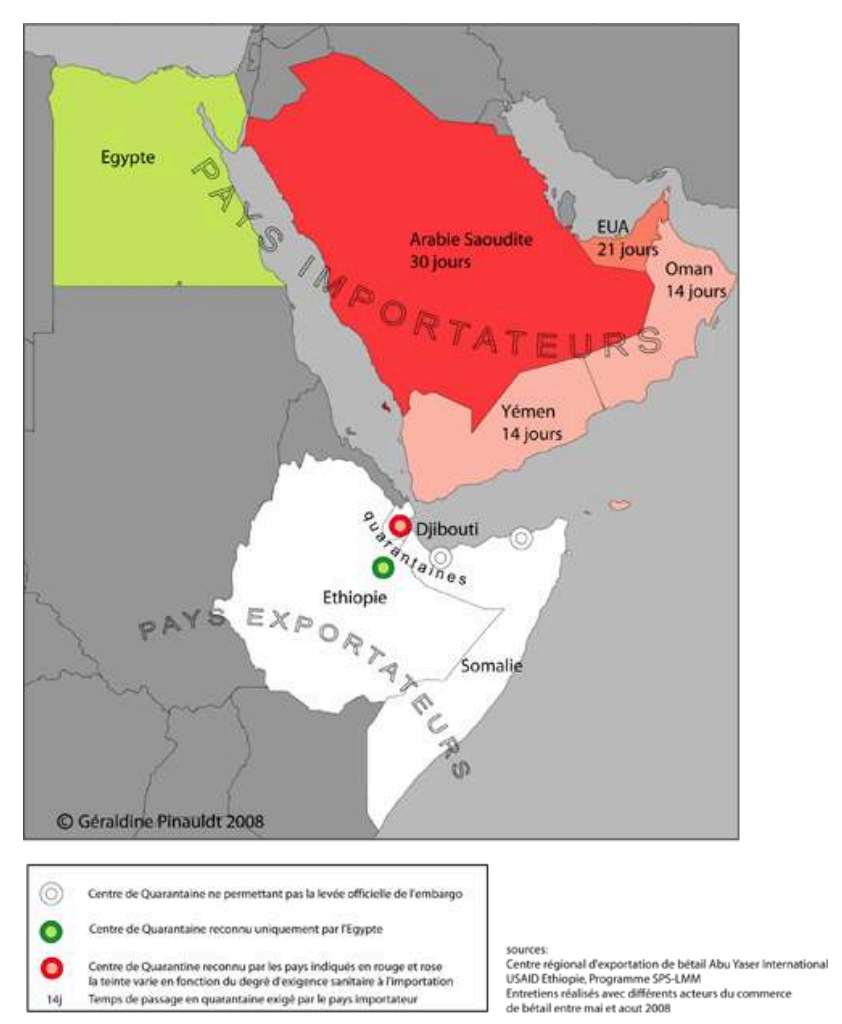

28

La décennie qui vient de s'écouler (1997/2007), a ainsi été très marquée par la fièvre de la vallée du Rift. La réorientation des flux ainsi que la course à la quarantaine qui s'en sont suivies ont été accompagnées d'une transformation des circuits commerciaux et, pour l'Éthiopie au moins, d'un investissement dans les équipements 
d'accompagnement autres que quarantaine. Ces transformations en cours permettent de mesurer à quel point un désastre sanitaire peut être vecteur de changement.

\section{La crise sanitaire, amorce d'une recomposition du système commercial ?}

29 A ce niveau de mon étude, les conséquences territoriales se dessinent mais n'apparaissent pas encore clairement. Néanmoins, la première mission de terrain effectuée en Éthiopie et à Djibouti m'a donné des indications d'une part sur la réorganisation des réseaux commerciaux, et d'autre part sur les transformations en terme d'équipement qui à terme peuvent avoir un impact fort sur les flux et les stratégies des pasteurs.

30 Alors que Marcel Djama (Bourgeot, 1999) a montré le passage, dans le système somali, d'un système articulé par les Abaan («big men») à celui qui prévaut actuellement et dont les dilaals (courtiers) constituent un pivot important, la conjonction de la mondialisation et des effets de la crise sanitaire semblent introduire un élément nouveau dans le système: la pénétration des marchands arabes dans l'arrière-pays somali et leur volonté d'un plus grand contrôle du marché en amont.

31 La réouverture du marché saoudien semble transformer les modes d'importation vers ce pays. En effet, auparavant, les importateurs arabes ne dépassaient jamais les ports d'exportation somali. En amont, la chaîne de commercialisation était gérée totalement par l'exportateur somali et sa cohorte d'intermédiaires, courtiers, transporteurs. Cette configuration est actuellement toujours dominante, mais il apparaît que de plus en plus, les commerçants arabes s'organisent pour pénétrer les territoires d'élevage, afin de maîtriser le commerce et de minimiser leurs risques.

En effet, les commerçants arabes sont soumis pour leur part à une forte concurrence, qui s'est accrue avec l'arrivée de nouveaux acteurs depuis les années 1980. Depuis 1983, la Banque Islamique de Développement (BID) a lancé le projet Adahi, qui consiste en l'achat de petits ruminants qui seront vendus dans le cadre de l'Aïd Al Adha, abattus par procuration, et dont la viande sera ensuite redistribuée comme aide alimentaire dans les pays musulmans démunis. Ayant commencé avec 63000 animaux achetés l'année du lancement, la Banque Islamique est aujourd'hui devenue le principal importateur de bétail de la Corne de l'Afrique, au travers d'entreprises privées d'importation (au nombre de 4), et fournit une grande part des moutons à sacrifier pendant le pèlerinage à la Mecque (700 000 à 800000 têtes importées par la BID pour les plus récents pèlerinages). Le reste du marché est partagé entre plusieurs acheteurs d'importance variable.

Ainsi, la tendance à la transformation des réseaux dans l'arrière-pays se fait selon deux types de configurations qui toutes deux prennent la forme d'une concurrence directe avec les exportateurs somali soit en créant des succursales locales dans les pays producteurs. Ce procédé permet d'accéder aux producteurs et aux petits marchés en utilisant les réseaux des agents locaux. Soit en négociant directement sur les marchés intermédiaires. Lors de mon séjour à Dire Dawa, j'ai rencontré un entrepreneur somali dont la spécialité n'est pas le bétail, qui avait récemment guidé un importateur saoudien dans la région. Ensemble, ils ont visité une demi douzaine de marchés afin de conclure des contrats mensuels d'approvisionnement, dans le but de réunir 36000 
moutons par an, ce qui le place parmi les importateurs moyens. Ceci illustre les nouvelles pratiques d'importation dans la compétition qui opposent les importateurs saoudiens, dont plusieurs, nouveaux dans la Corne de l'Afrique, opéraient auparavant en Europe de l'est. Ces pratiques avaient déjà été notées par V. Alary (2007).

On peut imaginer que ces nouvelles configurations peuvent aboutir à l'exclusion de certains marchands ou courtiers, et réorganiser en profondeur un système qui, à l'origine, ne mettait pas en concurrence directe Somali et Saoudiens, mais qui, dans un environnement naturel et commercial incertain (Scoones, 1999), est caractérisé par sa souplesse et l'opportunisme de ces acteurs. Si les acteurs du négoce sauront probablement adapter leurs stratégies dans ce contexte mouvant, il reste à définir l'impact de ces changements sur les éleveurs eux-mêmes.

Parallèlement à ces transformations, en Éthiopie, bien souvent avec l'appui de l'USAID, des investissements publics locaux sont effectués dans des infrastructures de marchés situés sur les routes d'exportations vers Djibouti ou le Somaliland. Certains petits marchés locaux pourraient ainsi avoir l'occasion de se transformer en nouvelle étape du commerce de bétail. On peut y voir également les prémices d'une nouvelle géographie des flux de l'exportation de bétail.

L'exemple de Shinile, en Éthiopie, illustre le rôle des infrastructures dans le contrôle du commerce de bétail. Cette ville située à $12 \mathrm{~km}$ de Dire Dawa, station du chemin de fer reliant Addis-Abeba à Djibouti, n'était à mon passage qu'un petit marché de petits ruminants, dont l'aire d'attraction pour les vendeurs se limitait à un rayon d'à peine une centaine de kilomètres et pour les acheteurs aux bouchères de la ville et à quelques commerçants de Dire-Dawa pour l'approvisionnement des boucheries de la ville. Il s'y vendait une cinquantaine de petits ruminants par jour (très variable selon la saison, entre 20 et 130). La municipalité et le district de Shinile ont fait construire un marché en dur destiné à accueillir des dromadaires et bovins. Afin de faire fonctionner à plein le marché, ils ont pris des contacts en amont et en aval du marché: en amont, afin d'approvisionner le marché, une association a été créée par la municipalité afin d'informer les vendeurs, mais dans un premier temps, la stratégie adoptée est d'investir à perte dans l'achat de gros bétail sur les marchés de Babille et Meisso. En aval, des contacts ont été pris avec des importateurs arabes présents à Djibouti afin de conclure des contrats directement avec eux. En juin 2008, le marché était construit et se préparait à être fonctionnel dans le mois qui suivait. L'idée de ce marché est née du projet d'amélioration de la route qui lie Addis Abeba à Djibouti sur le tracé du chemin de fer, plus courte que la route actuellement utilisée comme corridor d'accès à la mer. Il intervient dans un contexte où les actions des collectivités territoriales peuvent être instrumentalisées par l'État fédéral ou par l'État régional dans un contexte de partage du pouvoir local pas toujours bien défini. Il apparaît au travers de cet exemple que les pouvoirs publics à diverses échelles ont bien compris la manne que représentait l'exportation du bétail, et les opportunités de réorganisations dès lors que l'on se place à proximité de Djibouti et de son centre désormais régional d'exportation de bétail, et pas trop éloigné des autres routes.

Des projets similaires sont en cours dans d'autres localités le long du tracé du chemin de fer (et de la route dont la réfection devrait intervenir avec le projet de port sec de Dire Dawa), et de la route vers le Somaliland. Il sera intéressant d'évaluer l'impact de ces projets, et de vérifier plus à l'intérieur si les marchés sont l'objet de réorganisation 
et si une nouvelle hiérarchie se met en place dans la polarité de Djibouti ou encore selon d'autres logiques.

A un autre niveau, il est important de noter le passage de l'économie informelle à l'économie formelle d'un certain nombre d'acteurs en Éthiopie. Bien que n'ayant pas effectué d'enquête exhaustive, l'interprétation de discussions que j'ai pu mener avec certains acteurs est confirmée par les chiffres des revenus des exportations éthiopiennes. Par ailleurs, des entretiens avec certains exportateurs somali on mis en relief leur enthousiasme face à ces dynamiques et leur intérêt à plus ou moins long terme pour un type d'élevage en ranch.

39 A titre de rappel historique, on peut noter que ce ne serait pas la première fois qu'un embargo sanitaire aura suscité des transformations dans la région. En effet,: sous la menace d'un embargo américain sur les peaux et cuirs exportés du Somaliland lié à un risque d'anthrax, les Britanniques investissent pour la première fois dans des infrastructures liées à l'élevage au Somaliland en créant en 1924 le premier service vétérinaire du pays, ouvrant la voie à des aménagements tels que puits et zones de conservation, qui n'ont pas été sans effets sur la transformation alors en cours du pastoralisme et du commerce (Abdi Ismaïl Samatar, 1989). Les observations réalisées sur le terrain et présentées dans cette partie indiquent qu'en plus des jeux de quarantaines, les évènements de ces dernières années n'ont pas été sans conséquences. Il s'agit maintenant d'évaluer la pérennité de ces transformations.

\section{Conclusion}

Le risque d'une diffusion de la fièvre de la vallée du Rift hors d'Afrique a sonné le point de départ d'une recomposition de l'organisation de ce commerce entre Ethiopie, Somalie et Djibouti, entraînant des jeux d'acteurs qui commencent à avoir une traduction spatiale perceptible.

41 L'aire pastorale se trouvant placée sous différentes juridictions nationales, et le rôle des États semblant s'accroitre dans l'accompagnement de l'accès au marché des produits de l'élevage, il va être intéressant d'étudier les différentes formes que prendront ces reconfigurations, et les jeux d'acteurs sur des opportunités différenciées. La coordination des organisations internationales qui assistent les projets nationaux prend largement en compte la dimension transfrontalière de ce commerce, bien que, on l'a vu avec l'USAID, il peut y avoir des contradictions dans les actions d'un même organisme agissant au niveau régional.

La polarisation commerciale vers les pays du Golfe et la mise en place par ceux-ci de normes sanitaires strictes tend à une réorganisation originale côté africain. En effet, l'élevage était auparavant largement ignoré des discours nationaux sur le développement et absent des rapports interétatiques. Impliquant son entrée progressive dans la légalité, les mutations en cours s'accompagnent également d'une prise de conscience des bénéfices qu'il représente: financiers pour l'Éthiopie, en terme de positionnement régional pour Djibouti sur ces deux plans pour le Somaliland.

Sur un continent africain largement pastoral, cette activité a été placée en périphérie depuis la formation d'États coloniaux centrés sur les zones de production agricoles, provoquant, comme le suggère J.G Galaty (Bourgeot, 1999), une inévitable marginalisation de populations devenues transfrontalières. Ainsi, et malgré les 
particularités somali par rapport à ce modèle, les éléments présentés, parmi lesquels la progressive légalisation si elle se confirme, pourraient dessiner une reconfiguration inédite en Afrique des relations entre État, éleveurs et négociants de bétail.

\section{BIBLIOGRAPHIE}

Abdi Ismail Samatar, The State and Rural transformation in Northern Somalila, 1884-1986, the University of Wisconsin Press, 1989.

Djama M., Producteurs pastoraux et commerce international. L'évolution des rapports marchands en pays Nord-Somali in Bourgeot, Horizons nomades en Afrique Sahélienne. Sociétés, développement et démocratie, Karthala 1999.

Galaty, J. G. Les frontières pastorales en Afrique de l'Est, in Bourgeot, Horizons nomades en Afrique Sahélienne. Sociétés, développement et démocratie, Karthala 1999.

Ethiopian Customs Authority, Planning and research service, Towards modern customs by the new ethiopian millenium, Addis Abeba, nov. 2006.

Lewis I. M., Understanding Somalia and Somaliland, Hurst Publishers, 2008.

Scoones I (dir), Nouvelles orientations du développement pastoral en Afrique, vivre dans un environnement incertain, CTA-Karthala 1999.

\section{NOTES}

1. Les rivalités entre villes de la côte nord est africaine ne sont pas nouvelles, et, Zeyla, Tadjourah, Berbera, Djibouti ont tour à tour dominé le commerce des produits éthiopiens avec l'outre mer, et décliné.

i. La fièvre de la vallée du Rift est une zoonose virale qui affecte essentiellement le bétail mais peut se transmettre aux humains. Rapportée la première fois au Kenya en 1915, le virus n'a été isolé qu'en 1931.

ii. Les statistiques nationales éthiopiennes tiennent compte du calendrier éthiopien selon lequel l'année court de septembre à septembre, compliquant la comparaison des données nationales.

\section{RÉSUMÉS}

Depuis le XIX ${ }^{\mathrm{e}}$ siècle le territoire et la société nord somali se sont progressivement structurés en un système commercial dont les exportations de bétail vers les pays du golfe constituent l'élément principal. Au cours des années 1990, la conjonction des bouleversements politiques dans tous les pays de la région et d'un embargo sanitaire d'une ampleur sans précédent ont 
amorcé une transformation du système. Le principal port d'exportation pour le bétail, Berbera, dans l'actuel Somaliland, a perdu sa place au profit des ports de Djibouti et de Bosaaso au Puntland. La certification sanitaire, dont l'élément central est le centre de quarantaine associé à des services vétérinaires reconnus, constitue la clé de l'exportation vers les marchés de la péninsule arabe, et plus particulièrement l'important marché saoudien. Ainsi, les États participant à l'exportation de bétail se sont lancés dans une course à la quarantaine, qui fonctionne comme indicateur de transformations en cours à la fois dans les réseaux marchands et dans les lieux du commerce.

Livestock exports to the middle eastern countries, all along the $20^{\text {th }}$ century, have organised society and territory of the northern somali region. During the 90 's, this system has been challenged by both political turmoils in the whole horn of africa region, and a severe sanitary ban on livestock exports imposed by the middle eastern countries. Exports through Berbera, the former main livestock port, have seriously decreased, for the benefit of emerging Djibouti and Bosaaso. Sanitary certification, system in which quarantine station in association with recognized veterinary services is a core element, is today's only key to the middle eastern markets, especially to Saudi Arabia, the main importer. To get a chance to regain those lucrative markets, the States involved in livestock exports are trying to obtain recongnized quarantine stations. This «quarantine war » is the visible aspect of tranformations taking place in livestock market chain and in the territories of this trade.

\section{AUTEUR}

\section{GÉRALDINE PINAULDT}

Géraldine Pinauldt est doctorante à l'Institut Français de Géopolitique de l'Université de Paris 8. 